NOUVELLE

\section{Thérapie cellulaire de l'ischémie des membres inférieurs}

Joseph Emmerich
Service de Médecine

vasculaire et Inserm U.428,

Hôpital Européen Georges

Pompidou, 20, rue Leblanc,

75908 Paris Cedex 15,

France.

joseph.emmerich@

egp.ap-hop-paris.fr

rieure de la jambe. La même stratégie est également en cours d'étude dans la maladie coronaire, utilisant les mêmes gènes ou d'autres approches (pour revue, voir [7-12]). La seule étude effectuée en double aveugle contre placebo, une étude de phase II multicentrique européenne, dont le promoteur est le laboratoire Aventis (étude TALISMAN), a inclus 122 patients ayant une ischémie critique avec troubles trophiques. Elle utilise un plasmide nu, injecté par voie intramusculaire, codant pour le FGF-l. Ses résultats devraient être disponibles l'année prochaine.

\section{Thérapie cellulaire}

Dès 1997, le groupe de T. Asahara et J. Isner a mis en évidence dans la circulation des progéniteurs endothéliaux, qui, après isolement et amplification ex vivo, sont capables de participer à l'angiogenèse dans des modèles animaux d'ischémie [13]. Les progéniteurs des cellules endothéliales, exprimant l'antigène CD34 et d'origine médullaire, jouent un rôle majeur dans la vasculogenèse dans les conditions physiologiques ou pathologiques. La thérapie cellulaire au moyen de ces progéniteurs endothéliaux permet de stimuler l'angiogenèse dans des modèles animaux [14-17]. Elle présente plusieurs avantages par rapport à la thérapie génique décrite ci-dessus. Tout d'abord, ce sont des cellules autologues qui sont utilisées, prévenant ainsi tout risque de rejet. Elle élimine également le risque théorique de maladies induites 
par la thérapie génique, liées soit directement à l'action du facteur de croissance codé par le plasmide injecté, soit à une insertion du plasmide dans le génome, malgré toutes les précautions prises. Mais, comme la thérapie génique, la stimulation de l'angiogenèse par les progéniteurs endothéliaux peut induire des effets secondaires comme l'activation du développement de cancers latents, une accélération de l'athérosclérose ou d'une rétinopathie (notamment chez le diabétique). Jusqu'ici plus de 900 patients ayant une pathologie ischémique ont été traités par thérapie génique ou cellulaire sans effets secondaires authentifiés (pour revue, voir [18]).

Forte des résultats très prometteurs obtenus dans un modèle d'ischémie de la patte postérieure de rat nude après injection de progéniteurs endothéliaux isolés de sang de cordon [19], une équipe japonaise a réalisé une étude pilote chez l'homme [20]. La source de progéniteurs était une préparation autologue de cellules mononucléées issues de la moelle et la pathologie ciblée était l'ischémie critique des membres inférieurs. Un prélèvement de $500 \mathrm{ml}$ de moelle a été réalisé sous anesthésie générale, puis les cellules mononucléées ont été isolées et concentrées dans un volume final de $30 \mathrm{ml}$. Le nombre total de cellules injectées était compris entre 0,7 et 2,8 $810^{9}$. Au cours de l'étude pilote réalisée chez 25 patients ayant une ischémie d'un membre inférieur (groupe A), les cellules ont été administrées dans les trois heures suivant le prélèvement en 40 injections intramusculaires locales au niveau du membre ischémié, du sérum physiologique étant injecté dans l'autre membre. Compte tenu des résultats très positifs obtenus à l'issue de cette étude ouverte, un second groupe de patients a été inclus afin de réaliser une étude randomisée. Ce groupe (groupe B) était composé de 22 patients ayant une ischémie bilatérale des membres inférieurs. Les 44 membres inférieurs de ces 22 patients ont été randomisés et ont reçu des cellules mononu- cléées de la moelle osseuse ou des cellules mononucléées issues du sang périphérique (ne contenant pas de progéniteurs endothéliaux). Après inclusion, les patients ont été suivis pendant quatre semaines après greffe puis tous les quatre mois. Après quatre et 24 semaines, une amélioration significative de tous les critères cliniques ainsi que la formation de vaisseaux collatéraux objectivée par angiographie ont été observées dans tous les membres ayant reçu des cellules médullaires (mais pas dans le membre témoin, traité par cellules mononucléées du sang périphérique). Les ulcères et gangrènes ischémiques ont régressé de manière saisissante. Cette étude a montré pour la première fois l'efficacité et la sûreté de l'implantation de cellules mononucléées de moelle osseuse dans l'artérite des membres inférieurs. Deux autres études récentes, portant sur de plus faibles effectifs et utilisant le même protocole confirment la faisabilité de la thérapie cellulaire par cellules mononucléées isolées de la moelle osseuse [21, 22].

Une approche similaire avec injection intramyocardique ou intracoronaire a été effectuée dans l'ischémie myocardique chronique ou à la phase aiguë d'un infarctus du myocarde [18]. La seule étude randomisée dans l'infarctus du myocarde a été présentée récemment [23]. Dans cette étude de 60 patients, 30 sujets ont subi une intervention de revascularisation après leur infarctus et ont reçu 4 jours plus tard, par voie intracoronaire, des cellules mononucléées médullaires; 30 autres patients n'ont pas subi cette phase de thérapie cellulaire. Une amélioration significative à 6 mois de la fonction ventriculaire gauche a été observée, sans effet délétère. L'innocuité de l'injection intracoronaire de cellules médullaires a été remise en question récemment dans deux travaux. L'un montre que les cellules peuvent induire un infarctus du myocarde dans un modèle canin [24], et l'autre montre que l'injection de cellules souches prélevées après injection de G-CSF (granulo- cyte colony-stimulating factor) induit une resténose coronaire fréquente [25]. Cette stratégie d'angiogenèse thérapeutique constitue un espoir thérapeutique important pour de nombreux malades actuellement dans une impasse thérapeutique. Une optimisation du protocole de thérapie cellulaire après isolement et expansion des progéniteurs responsables de cette angiogenèse thérapeutique serait un important progrès, qui permettrait d'alléger la procédure thérapeutique et notamment de s'affranchir d'une anesthésie générale et d'un prélèvement médullaire important. De nombreuses questions restent en suspens concernant cette approche thérapeutique de l'ischémie critique des membres inférieurs non revascularisables.

- Quel type cellulaire utiliser? Cellules mononucléées médullaires totales, progéniteurs endothéliaux circulants $\mathrm{CD} 34^{+}$, autres cellules?

- Quelle est la meilleure voie d'administration? Intramusculaire, intra-artérielle?

- Quel nombre optimal de cellules faut-il administrer?

- Faut-il répéter les injections et si oui avec quelle fréquence?

- Existe-il une toxicité à moyen et à long terme de ce traitement?

- Nécessité d'essais en double aveugle pour prouver l'efficacité de cette stratégie?

Malgré toutes ces questions, cette approche thérapeutique qui n'en est qu'à ses prémices représente un espoir important pour les patients chez lesquels à l'heure actuelle l'ultime recours est encore trop souvent l'amputation. Elle soulève également de nombreuses questions telles que les mécanismes d'action des cellules injectées: l'efficacité estelle due à une vasculogenèse ou à un effet paracrine stimulant localement l'angiogenèse? Quels sont les mécanismes d'adressage? Peut-on améliorer la vectorisation cellulaire? Et, enfin, peut-on aussi utiliser ces cellules comme vecteur thérapeutique dans d'autres maladies? $\diamond$ Cell therapy in ischemia 


\section{RÉFÉRENCES}

1. Dormandy JA, Rutherford, RB. Management of peripheral arterial disease (PAD). TransAtlantic inter-society consensus (TASC) working group. J Vasc Surg 2000; 31: S1-296.

2. The ICAI Group. Long-term mortality and its predictors in patients with critical leg ischaemia. The study group of criticial chronic ischemia of the lower extremities. Eur J Vasc Endovasc Surg 1997; 14: 91-5.

3. Isner JM, Pieckzek A, Schainfeld R, et al. Clinical evidence of angiogenesis after arterial gene transfer of phVEGFl65 in patients with ischaemic limb. Lancet 1996; 348: 370-4.

4. Baumgartner I, Pieckzek A, Manor 0. Constitutive expression of phVEGF165 after intramuscular gene transfer promotes collateral vessel development in patients with critical leg ischemia. Circulation 1998; 97:1114-23.

5. Shyu KG, Chang H, Wang BW, Kuan P. Intramuscular vascular endothelial growth factor gene therapy in patients with chronic critical leg ischemia Am J Med 2003; 114: 85-92.

6. Comerota AJ, Throm RC, Miller KA, et al. Naked plasmid DNA encoding fibroblast growth factor type 1 for the treatment of end-stage unreconstructible lower extremity ischemia: preliminary results of a phase I trial. J Vasc Surg 2002; 35: 930-6.

7. Freedman SB, Isner JM. Therapeutic angiogenesis for coronary artery disease. Ann Intern Med 2002; 136: 54-71.

8. Losordo DW, Dimmeler S. Therapeutic angiogenesis and veasculogenesis for ischemic disease. Part I: angiogenic cytokines. Circulation 2004; 109: 2487-91.

9. Krais T, Muller B. Iloprost in pheripheral vascular disease. Cardiovasc Drug Rev 1991; 9: 158-71.

10. Lederman RJ, Mendelsohn F0, Anderson RD, et al. Therapeutic angiogenesis with recombinant fibroblast growth factor-2 for intermittent claudication (the TRAFFIC study): a randomized trial. Lancet 2002; 359: 2053-8.

11. Takeshita S, Isshiki T, Ochiai M, et al. Endotheliumdependent relaxation of collaterals micorvessels after intramuscular gene transfer of vascular endothelium growth factor in a rat model of hindlimb ischemia. Circulation 1998; 98: 1261-3.

12. Tsurimi $Y$, Takeshita $S$, Chen D, et al. Direct intramuscular gene transfer of naked DNA encoding vascular endothelial growth factor augments collateral development and tissue perfusion. Circulation 1996; 94: 3281-90.

13. Asahara T, Murohara T, Sullivan A, et al. Isolation of putative progenitor endothelial cells for angiogenesis. Science 1997; 275: 964-7.

14. Asahara T, Masuda H, Takahashi T, et al. Bone marrow origin of endothelial progenitor cells responsible for postnatal vasculogenesis in physiological and pathological neovascularization. Circ Res 1999; 85: 221-8.

15. Kalka C, Masuda H, Takahashi T, et al. Transplantation of ex vivo expanded endothelial progenitor cells for therapeutic neovascularization Proc Natl Acad Sci USA 2000; 97 : 3422-7.

16. Murohara T, Ikeda H, Duan J, et al. Transplanted cord blood-derived endothelial precursor cells augment postnatal neovascularization. J Clin Invest 2000; 105: 1527-36.

17. Shintani S, Murohara T, Ikeda H, et al. Augmentation of postnatal neovascularization with autologous bone marrow transplantation. Circulation 2001; 103: 897-95.

18. Losordo DW, Dimmeler S. Therapeutic angiogenesis and vasculogenesis for ischemic disease. Part II: cell based therapies. Circulation 2004; 109: 2692-7.

19. Iba 0 , Matsubara H, Nozawa Y, et al. Angiogenesis by implantation of peripheral blood mononuclear cells and platelets into ischemic limbs. Circulation 2002; 106: 2019-25.

20. Tateishi-Yuyama E, Matsubara H, Murohara T, et al. Therapeutic angiogenesis for patients with limb ischaemia by autologous transplantation of bonemarrow cells: a pilot study and a randomised controlled trial. Lancet 2002; 360: 427-35.

21. Higashi Y, Kimura M, Hara K, et al. Autologous bone-marrow mononuclear cell implantation improves endothelium-dependent vasodilation in patients with limb ischemia. Circulation 2004; 109: 1215-8

22. Huang PP, Li SZ, Han MZ, et al. Autologous transplantation of peripheral blood stem cells as an effective therapeutic approach for severe arteriosclerosis obliterans of lower extremities. Thromb Haemost 2004; 91: 606-9.

23. Wollert KC, Meyer GP, Lotz J, et al. Randomized controlled clinical trial of intracoronary autologous bone marrow cell transfer post myocardial infarction. Circulation 2003; 108: 2723 (abstract).

24. Vulliet PR, Greeley M, Halloran SM, et al. Intracoronary arterial injection of mesenchymal stromal cells and microinfarction in dogs. Lancet 2004; 363 : 783-4.

25. Kang HJ, Kim HS, Zhang SY, et al. Effects of intracoronary infusion of peripheral blood stemcells mobilised with granulocyte-colony stimulating factor on left ventricular systolic function and restenosis after coronary stenting in myocardial infarction: the MAGIC cell randomised clinical trial. Lancet 2004 ; 363: 751-6.

\section{NOUVELLE}

\section{Identification d'un nouveau gène de susceptibilité à la lèpre par clonage positionnel}

Alexandre Alcaïs, Laurent Abel
Laboratoire de Génétique

humaine des maladies

infectieuses,

Université René Descartes, Inserm U.550,

Faculté de Médecine Necker, 156 , rue de Vaugirard, 75015 Paris, France. alcais@necker.fr
> La lèpre, ou maladie de Hansen, est une maladie infectieuse chronique, provoquée par le bacille Mycobacterium leprae, qui atteint principalement les parties les plus froides du corps, en particulier la peau, les segments superficiels des nerfs périphériques, les voies respiratoires supérieures, les testicules et les yeux [1]. La lèpre est, parmi les maladies transmissibles, celle qui cause le plus d'incapacités physiques permanentes, et les difformités terribles dues à la forme avancée de la maladie ont largement contribué aux préjugés pro- fonds à l'origine du rejet des malades et de leurs proches par la société. Heureusement, les avancées thérapeutiques décisives des années 1980 ont conduit, en 1991, l'Organisation Mondiale de la Santé à faire de l'élimination de la lèpre un de ses objectifs majeurs pour la fin de l'année 2000. De fait, parmi les 122 pays considérés comme endémiques en 1985, 108 ont effectivement éradiqué la maladie, et c'est au total plus de 12 millions de patients qui ont été guéris grâce à la polychimiothérapie [2]. Toutefois, parodiant Mark Twain, on peut se demander si «les rumeurs sur la disparition de la lèpre n'ont pas été grandement exagérées». En effet, la polychimiothérapie n'a pas eu d'impact sur le cycle de transmission de la maladie et tandis que l'on diagnostiquait environ 600000 nouveaux cas de lèpre en 1991, ce sont 760000 nouveaux cas qui ont été dépistés dans le monde en 2001. La cause de la stagnation de cette incidence à un niveau élevé, alors que le réservoir naturel de $M$. leprae est limité à I'homme et le traitement efficace, n'est pas connue, indiquant que des aspects importants de la relation complexe entre 